\title{
MBD2 Regulates Th17 Cell Differentiation and Experimental Severe Asthma by Affecting IRF4 Expression
}

\author{
Aijun Jia, ${ }^{1,2}$ Yueling Wang, ${ }^{3}$ Wenjin Sun, ${ }^{1}$ Bing Xiao, ${ }^{2}$ Yan Wei, ${ }^{4}$ Lulu Qiu, ${ }^{1}$ Lin Mu, ${ }^{5}$ Li Xu, ${ }^{6}$ \\ Jianmin Li, ${ }^{7}$ Xiufeng Zhang, ${ }^{8}$ Da Liu, ${ }^{9}$ Cong Peng, ${ }^{10}$ Dongshan Zhang, ${ }^{2}$ and Xudong Xiang ${ }^{2}$ \\ ${ }^{1}$ Department of Respiratory Medicine, Hunan Centre for Evidence-Based Medicine, Research Unit of Respiratory Diseases, \\ The Second Xiangya Hospital, Central South University, 139 Middle Renmin Road, Changsha, Hunan 410011, China \\ ${ }^{2}$ Department of Emergency, Institute of Emergency Medicine and Difficult Diseases, The Second Xiangya Hospital, Central South \\ University, 139 Middle Renmin Road, Changsha, Hunan 410011, China \\ ${ }^{3}$ Department of Anesthesiology, Xiangya Hospital, Central South University, 87 Xiangya Road, Changsha, Hunan 410008, China \\ ${ }^{4}$ Department of Respiratory Medicine, The First Hospital of Guangyuan City, 490 Juguo Road, Guangyuan, Sichuan 628000, China \\ ${ }^{5}$ Department of Respiratory Medicine, Peace Hospital, Changzhi Medical College, Changzhi, Shanxi 046000, China \\ ${ }^{6}$ Department of the Second Thoracic Medicine, The Affiliated Cancer Hospital of Xiangya School of Medicine, Central South \\ University, 283 Tongzipo Road, Changsha, Hunan 410006, China \\ ${ }^{7}$ Department of Respiratory Medicine, Hunan Provincial People's Hospital, 61 West Jiefang Road, Changsha, Hunan 410005, China \\ ${ }^{8}$ Department of Respiratory Medicine, The Second Hospital, University of South China, 30 Jiefang Road, Hengyang, \\ Hunan 421001, China \\ ${ }^{9}$ Department of Respiratory Medicine, Changsha Central Hospital, 161 South Shaoshan Road, Changsha, Hunan 410004, China \\ ${ }^{10}$ Department of Dermatology and Venereology, Xiangya Hospital, Central South University, 87 Xiangya Road, Changsha, \\ Hunan 410008, China
}

Correspondence should be addressed to Xudong Xiang; xudongx_99@hotmail.com

Received 17 April 2017; Accepted 31 May 2017; Published 20 July 2017

Academic Editor: Younghyo Kim

Copyright @ 2017 Aijun Jia et al. This is an open access article distributed under the Creative Commons Attribution License, which permits unrestricted use, distribution, and reproduction in any medium, provided the original work is properly cited.

Th17 cells and IL-17 participate in airway neutrophil infiltration characteristics in the pathogenesis of severe asthma. Methyl-CpG binding domain protein 2 (MBD2) expression increased in $\mathrm{CD}^{+} \mathrm{T}$ cells in peripheral blood samples of asthma patients. However, little is known about that epigenetic regulation of MBD2 in both immunological pathogenesis of experimental severe asthma and $\mathrm{CD}^{+}{ }^{+} \mathrm{T}$ cell differentiation. Here, we established a neutrophil-predominant severe asthma model, which was characterized by airway hyperresponsiveness (AHR), BALF neutrophil granulocyte (NEU) increase, higher NEU and IL-17 protein levels, and more Th17 cell differentiation. In the model, MBD2 and IRF4 protein expression increased in the lung and spleen cells. Under overexpression or silencing of the MBD2 and IRF4 gene, the differentiation of Th17 cells and IL-17 secretion showed positive changes. IRF4 protein expression showed a positive change with overexpression or silencing of the MBD2 gene, whereas there was no significant difference in the expression of MBD2 under overexpression or silencing of the IRF4 gene. These data provide novel insights into epigenetic regulation of severe asthma.

\section{Introduction}

Asthma is a complex chronic inflammatory disease characterized by AHR, reversible airflow obstruction, and airway inflammation [1]. IL-17 (also known as IL-17A) is a representative cytokine produced by Th17 cells, which can be induced by the release of IL-8, CXC, and other neutrophil chemokines to raise and activate neutrophils [2-4], and animal and clinical analysis of samples have proved that Th17 cells and IL-17 play important roles in the pathogenesis of asthma. These asthma cases are characterized by predominantly neutrophilic and mixed granulocytic types and are associated with severe asthma and poor response to corticosteroids [5-9]. It is therefore very important to establish a 
NEU predominant inflammatory phenotype asthma model, and we tried using $100 \mu \mathrm{g}$ of HDM and OVA combined with $15 \mu \mathrm{g}$ LPS to establish a neutrophil-predominant severe asthma model and to further study the relationship between the changes of Th17 cells and the epigenetic alterations.

MBD2 (methyl-CpG binding domain protein 2) can specifically bind to the promoter region of a target gene and change in the posttranscriptional modification of histone through the recruitment of other molecules, thus changing the chromatin structure and regulating the expression of target genes. In our preliminary study, we found that the expression of MBD2 increased obviously after accepting stimulus differentiation in splenic $\mathrm{CD}^{+} \mathrm{T}$ cell in mice. Through the analysis of peripheral blood samples of asthma patients, we also found that MBD2 expression increased in $\mathrm{CD}^{+} \mathrm{T}$ cells in peripheral blood compared with healthy people, meaning that MBD2 has a close relationship with both the immunological pathogenesis of asthma and $\mathrm{CD} 4^{+} \mathrm{T}$ cell differentiation. Therefore, MBD2 could play an important role in neutrophil-predominant severe asthma, and to find which cytokine it impacts is our next research goal.

IRF4 (interferon regulatory factor 4) is strictly expressed in immune cells. In IRF4 research on the role of Th17 cell differentiation, Brüstle found that mice knockout IRF4 failed to develop experimental autoimmune encephalomyelitis, $\mathrm{T}$ cell and RORgamma T were expressed lower, and Th17 cell differentiation was impaired [10]. So, IRF4 is therefore an important factor in neutrophil-predominant severe asthma.

At present, there is little research on the role of MBD2 in severe asthma, and the relationship between MBD2 and IRF4 in severe asthma is not known. Here, through the above data review, we hypothesize that MBD2 would regulate Th17 cell differentiation and experimental severe asthma by affecting IRF4.

\section{Materials and Methods}

2.1. Ethics Statement. All studies were performed in compliance with the Second Xiangya Hospital and Central South University Animal Care and Use Committee guidelines.

2.2. Mice. Female C57BL/6 mice (provided by animal center of the Second Xiangya hospital of Central South University, China), aged 6-7 weeks, weighing 18-20 grams, were used in the experiments. All mice were bred and housed in a SPF facility with a 12/12 h light/dark cycle.

\subsection{Experimental Reagents and Equipment. The HDM were} supplied by GREER; the LPS, aluminum hydroxide gel, and OVA were supplied by Sigma; small animal cough- and asthma-inducing instrument (YLS-8A) was purchased from Beijing Zhongshidichuang Science and Technology Development Co. Ltd.; mice spirometer (MAX 1320) was purchased from Buxco $^{\circledR}$, USA; low-speed centrifuge (5810 R) was purchased from Eppendorf ${ }^{\circledR}$, Germany; optical microscope (DMI3000B) was from Olympus, Japan (Leica ${ }^{\circledR}$ ); and pathological image analyzer (Leica Application Suite V4) was purchased from Leica, Germany.
2.4. Mice Asthma Model. Female C57BL/6 mice were grouped according to a random number table, with six mice in each group. The group of severe asthma model mice were given an intraperitoneal sensitization injection with $100 \mu \mathrm{g}$ $\mathrm{HDM}+100 \mu \mathrm{g}$ OVA+ $15 \mu \mathrm{g}$ LPS $+2 \mathrm{mg}$ aluminum hydroxide on days 0,1 , and 2 [11] and then challenged with OVA solution atomized for 30 minutes before HDM intranasal excitation on days $14,15,18$, and 19 . For the saline group, mice received saline only. The group of conventional asthma model mice were given a sensitization intraperitoneal injection with $25 \mu \mathrm{g}$ OVA $+1 \mathrm{mg}$ aluminum hydroxide on days 0 and 7 and then challenged with OVA solution atomized excitation for 30 minutes on days $14,15,16,17,18,19$, and 20 [12]. Mice were sacrificed on day 21.

2.5. Ethology Observed. Mice ethology was observed everyday as follows: fur luster, nose and ear scratching, irritability, sneezing, rapid breathing, and incontinence.

2.6. Airway AHR. Methacholine- (Mch-) induced airway resistance was measured on day 21 ( $24 \mathrm{~h}$ after the final challenge) by direct plethysmography (Buxco Electronics, RC System, USA). The procedures were the same as previously described [13]. Mice were anesthetized, tracheotomized, and then intubed. At first, the baseline of lung resistance (RL0) was recorded for one minute. Then, mice were given $10 \mu \mathrm{l}$ saline and $10 \mu \mathrm{l}$ Mch with increasing doses of $0.39 \mathrm{mg} / \mathrm{ml}$ (dose 1 ), $0.78 \mathrm{mg} / \mathrm{ml}$ (dose 2), $1.56 \mathrm{mg} / \mathrm{ml}$ (dose 3 ), and $3.12 \mathrm{mg} / \mathrm{ml}$ (dose 4) atomized to stimulate the airway, and the changes of lung resistance (RLX) were then recorded. The ratios of RLX/RL0 were used as the final results for analysis.

2.7. BALF Cell Count. BALF collection procedures were the same as previously described [13]. After eliminating red blood cells, by centrifugation and precipitation, total BALF cell counts were determined by a haemocytometer. Then, cell slices were made using a Biping settlement system. BALF NEU and EOS cell counts were determined in 200 total BALF cells after cell slices underwent H\&E staining.

2.8. Histopathological Analysis of Inflammatory and Structural Changes. The right lungs were incubated in $4 \%$ paraformaldehyde for $24 \mathrm{~h}$ and level dehydration: $70 \%$ ethanol ( $5 \mathrm{~min}$ ), $75 \%$ ethanol ( $5 \mathrm{~min}$ ), $80 \%$ ethanol ( $5 \mathrm{~min}$ ), $90 \%$ ethanol ( $5 \mathrm{~min}), 95 \%$ ethanol $(5 \mathrm{~min}), 100 \%$ ethanol I (10 min), 100\% ethanol II (10 min), xylene liquid I (10 min), and xylene liquid II (10 min) and then embedded in paraffin.

2.8.1. Lung Tissue Inflammation Score. Lung tissues were stained with $\mathrm{H} \& \mathrm{E}$, and $3 \sim 4 \mathrm{H} \& \mathrm{E}$ stained sections were chosen per group under a single-blind procedure. Two pathologists assessed the lung tissue inflammation score under a microscope as previously described [14]: a score of 0 represented no inflammatory cell infiltration; a score of 1 represented little inflammatory cell infiltration; a score of 2 represented 1 layer of inflammatory cells around the airway; a score of 3 represented 2 4 layers of inflammatory cells around the airway; and a score of 4 represented 4 or more layers of inflammatory cells around the airway. 
2.8.2. Immunohistochemistry for NEU, EOS, IL-17A, IL-4, $M B D 2$, and IRF4. Lung tissues were stained for immunohistochemistry (neutrophil-specific antibody (anti-Gr1, Biolegend), eosinophil antibody (anti-ECP, Biorbyt), IL-17A antibody (Proteintech), IL-4 antibody (ABBIOTEC), MBD2 antibody (Abcam), and IRF4 antibody (Proteintech)), and 2 4 H\&E stained sections per group were chosen under single-blind conditions to detect and localize NEU, EOS, IL-17A, IL-4, MBD2, and IRF4 protein expression. Using a microscope and computer image processing system (ImagePro Plus 6.0), two pathologists assessed the NEU, EOS, IL17A, IL-4, MBD2, and IRF4 scores.

2.9. Western Blotting. Total proteins were prepared using RIPA lysis buffer supplemented with protease inhibitors. Western blotting was carried out by probing the membranes with indicated primary antibodies followed by incubating with an HRP-conjugated secondary antibody. The NEU, EOS, IL-17A, IL-4, MBD2, and IRF4 antibodies were the same as above.

2.10. Bronchial Lung Tissue Suspension Cells. Mice bronchial lungs were collected, washed once with $5 \mathrm{x}$ antibiotic, and washed 2 times with PBS. Then, the lungs were cut as little as possible, and subsequently, digestive juice consisting of collagenase $1(0.5 \mathrm{mg} / \mathrm{ml}$ Sigma, unit $/ \mathrm{ml})+10 \mu \mathrm{g} / \mathrm{ml}$ DNase in RPMI medium was added and kept for 1 hour at $37^{\circ} \mathrm{C}$ in a water bath shaking incubator. After the digestion of the lung tissue and straining with a $70 \mu \mathrm{m}$ filter Cell Strainer (BD Falcon), cells were centrifugated and resuspended in $10 \%$ FBS culture medium.

2.11. T Cell Purification, Activation, and Staining. Spleen $\mathrm{CD}^{+} \mathrm{T}$ cells of model mice were selected by using microbead sorting (130-049-201, Miltenyi Biotec, Germany) and were then seeded in 12-well flat bottom plates. In the next 5 hours, the cells were restimulated with $50 \mathrm{ng} / \mathrm{ml}$ phorbol-12-myristate-13-acetate (PMA) (Multi Sciences Company, China), $1 \mu \mathrm{g} / \mathrm{ml}$ ionomycin (Multi Sciences Company, China), and $3 \mu \mathrm{g} / \mathrm{ml}$ monensin (Multi Sciences Company, China). The lung cells were then stained for surface marker FITCantiCD4 cytokine antibody (BioLend, USA) followed by fixation and permeabilization with fixation and permeabilization buffer (Multi Sciences Company, China) 15 min. After washing with permeabilization buffer, the lung and spleen cells were stained with intracellular markers APCanti-IL-17 and PE-anti-IL-4 cytokine antibodies (BioLend, USA) in the permeabilization buffer for $20 \mathrm{~min}$. Flow cytometry was conducted and the data were analyzed using the FACSCalibur and FlowJo version X software.

2.12. Th17 Cell Differentiation. For directed differentiation of Th17 cells, $1 \times 10^{6} \mathrm{CD}^{+} \mathrm{T}$ cells from C57BL/6 mice were activated with $2 \mu \mathrm{g} / \mathrm{ml}$ anti-CD3, $2 \mu \mathrm{g} / \mathrm{ml}$ anti-CD28 for 3 days in the presence of $5 \mathrm{ng} / \mathrm{ml}$ TGF-beta, $20 \mathrm{ng} / \mathrm{ml} \mathrm{IL-6}$ and $20 \mathrm{ng} / \mathrm{ml} \mathrm{IL-23.} \mathrm{For} \mathrm{neutralization} \mathrm{of} \mathrm{IL-4} \mathrm{and} \mathrm{IFN-}$ gamma, $10 \mu \mathrm{g} / \mathrm{ml}$ anti-IL-4 and $10 \mu \mathrm{g} / \mathrm{ml}$ anti-INF-gamma were added into the cultures $[15,16]$. All cytokines were purchased from BioLend Co. (BioLend, USA).
2.13. Lentiviral Transduction of Th17 Cells. Splenic CD $4^{+}$ Th17 lymphocytes were transfected with chemosynthesis MBD2 siRNA sequence (S) 5'-GTTTGGCTTAACACA TCTCAA-3' and IRF4 siRNA sequence (S) 5'-GCCA GACAACTGTATTACTTT-3'. The Th17 cells were cultured in 1640 medium without serum. The cells were resuspended according to $1.5 \times 10^{6} /$ tube and MOI $=20$, and the appropriate amount of virus was used for transfection. The cells were seeded in 12-well flat-bottom plates for $72 \mathrm{~h}$. In the last $5 \mathrm{~h}$, the cells were restimulated with PMA, ionomycin, and monensin as above.

\section{Results}

3.1. The Severe Asthma Mice Model Was Established. We first observed sleepiness in severe asthma mice on the 4th day of stimulation, compared to the 5th day for conventional asthma mice. When Mch was inhaled, the pulmonary resistance (RL) of the severe group showed a sharp rise (Figure 1(a)). Through BALF of the three groups, we found that total cell counts and neutrophil granulocyte counts of the severe group were the highest, but the eosinophil granulocyte counts of the conventional group were the most (Figure 1(b)). Histological analyses of lungs from the severe group exhibited markedly enhanced peribronchial inflammation with infiltrated neutrophils, but eosinophils were not obviously different compared with the conventional group (Figure 1(c)). Results of Western blot analyses were the same as those of histological analyses (Figure 1(d)).

3.2. The Severe Asthma Mice Was Mediated by Th17 Cells. IL-17 is the main representative cell factor of Th17 cells, as is IL-4 for Th2 cells. Histological analyses of lungs from the severe group exhibited markedly enhanced cells with stained IL-17, but cells with stained IL-4 were not obviously different compared with the conventional group (Figure 2(a)). Results of Western blot analyses were the same as histological analyses (Figure 2(b)). Th17 cells were the most in bronchial lung tissue suspension cells and splenocytes from the severe group compared with the conventional group (Figure 2(c)).

3.3. Expression of MBD2 in Severe Asthma Mice. Histological analyses of lungs from the severe group exhibited markedly enhanced cells with stained MBD2 compared with the conventional group (Figure 3(a)). MBD2 expression in lungs and splenic $\mathrm{CD} 4^{+} \mathrm{T}$ cells from the severe group were significantly increased compared with the conventional group (Figures 3(b) and 3(c)).

3.4. IL-17 Expression and Th17 Cell Differentiation under MBD2 Gene Silencing or Overexpression. We conducted Western blot analyses and demonstrated either MBD2 gene silencing $(\mathrm{M}(-))$ or overexpression $(\mathrm{M}(+))$ in splenic $\mathrm{CD}^{+} \mathrm{T}$ cells successfully. With MBD2 gene silencing, IL-17 expression was significantly lower than that of the empty transfection group $(\mathrm{M}(0))$; and when the MBD2 gene was overexpressed, IL-17 expression was markedly increased compared to that of the empty transfection group (Figure 4(a)). Th17 cells were obviously decreased or increased under MBD2 gene silencing or overexpression (Figure 4(b)). 


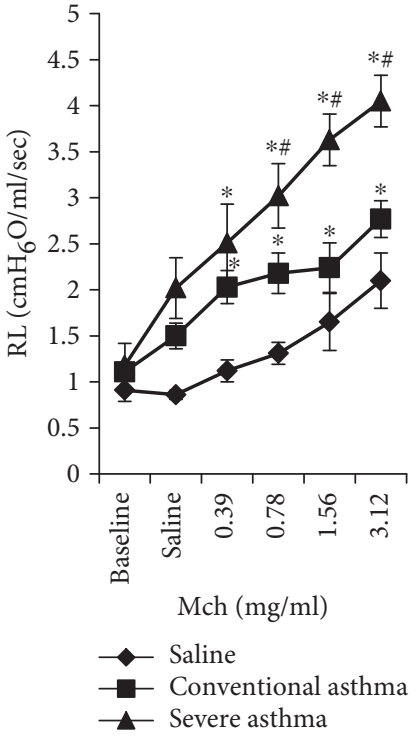

(a)

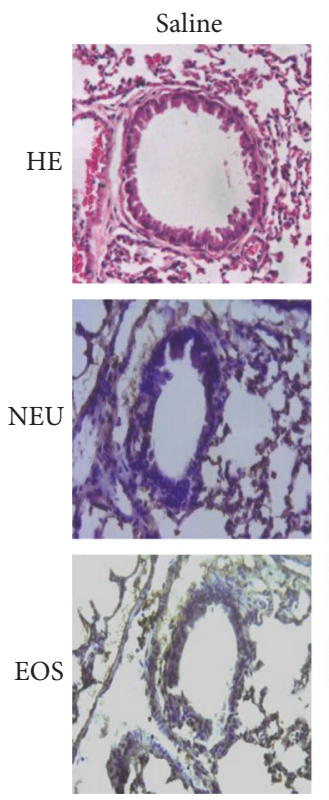

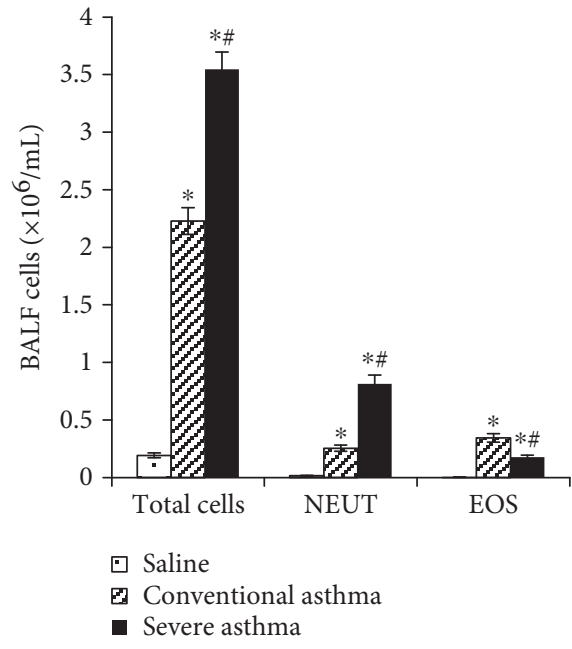

(b)
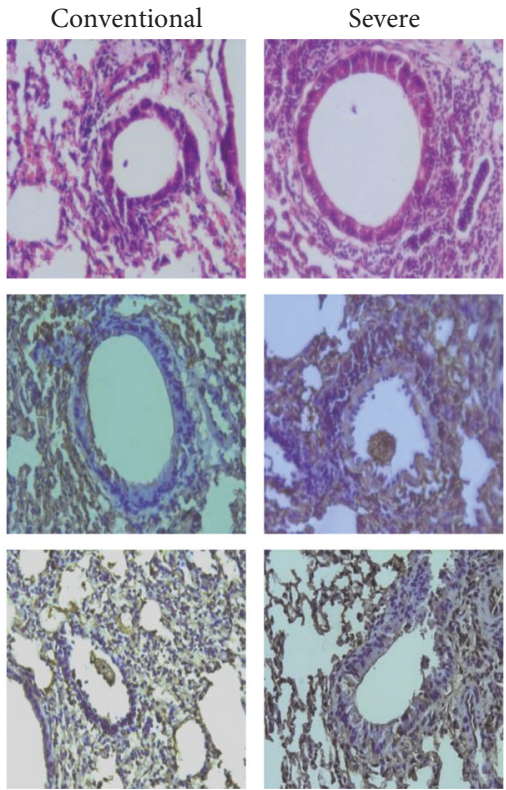

(c)

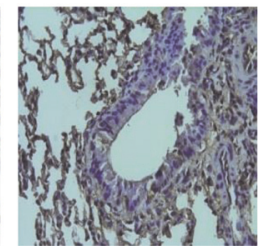

-

Saline Conventional Severe

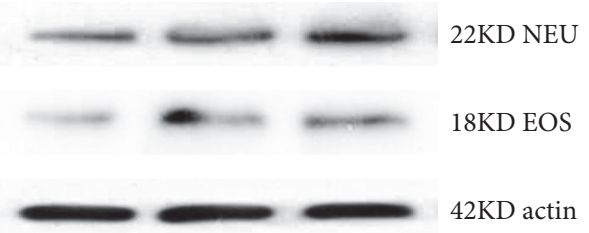

(d)

FIGURE 1: The severe asthma mice model was established. (a) Pulmonary resistance in the saline, conventional, and severe groups. When Mch was inhaled, the RL of the conventional and severe groups showed a rise, and the RL values of the severe group were higher than those of the conventional group. (b) The total, NEU, and EOS cells of BALF from the three groups. The total and NEU cells of BALF from the severe group were higher than those from the conventional group, but EOS cells showed an opposite result. (c) Lung tissues were stained with H\&E, immunohistochemistry (neutrophil-specific antibody (anti-Gr1), eosinophil antibody (anti-ECP)) of the three groups. Histological analyses of lungs from the severe group exhibited markedly enhanced peribronchial inflammation with infiltrated neutrophils, but eosinophils were not obviously different compared with the conventional group. (d) Western blot analysis detected NEU and EOS protein expression in the three groups. The NEU protein expression of the lungs from the severe group were higher than those from the conventional group, whereas EOS protein expression values were not obviously different between them. ${ }^{*} p<0.05$ as compared with the control group. ${ }^{*} p<0.05$ as compared with the conventional group. 


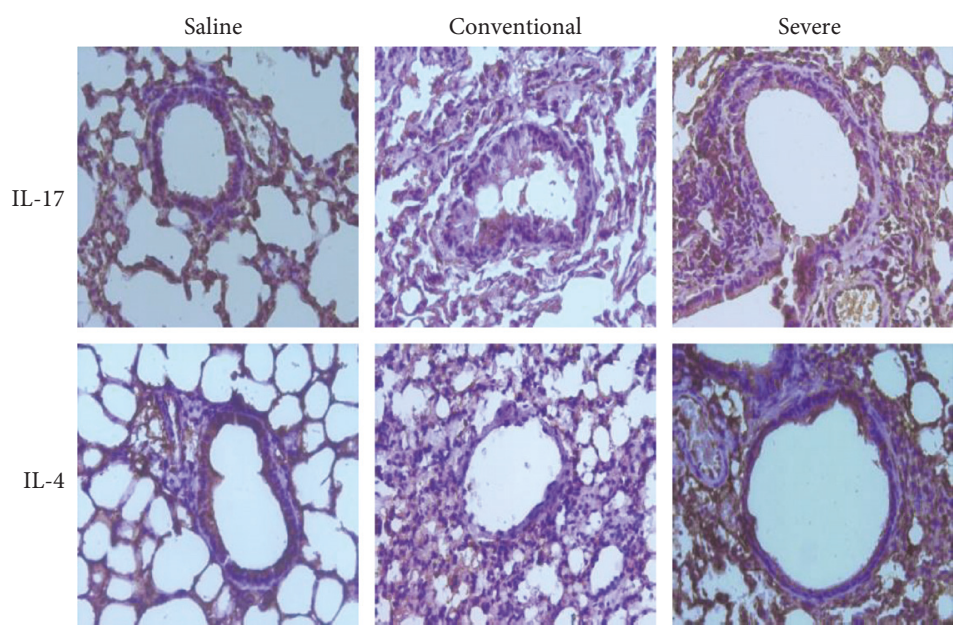

(a)

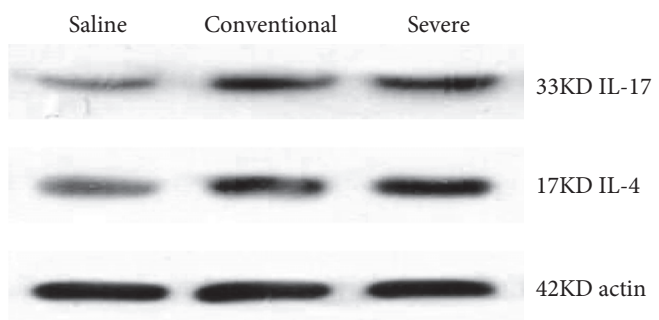

(b)

Bronchial lung tissure suspension cells
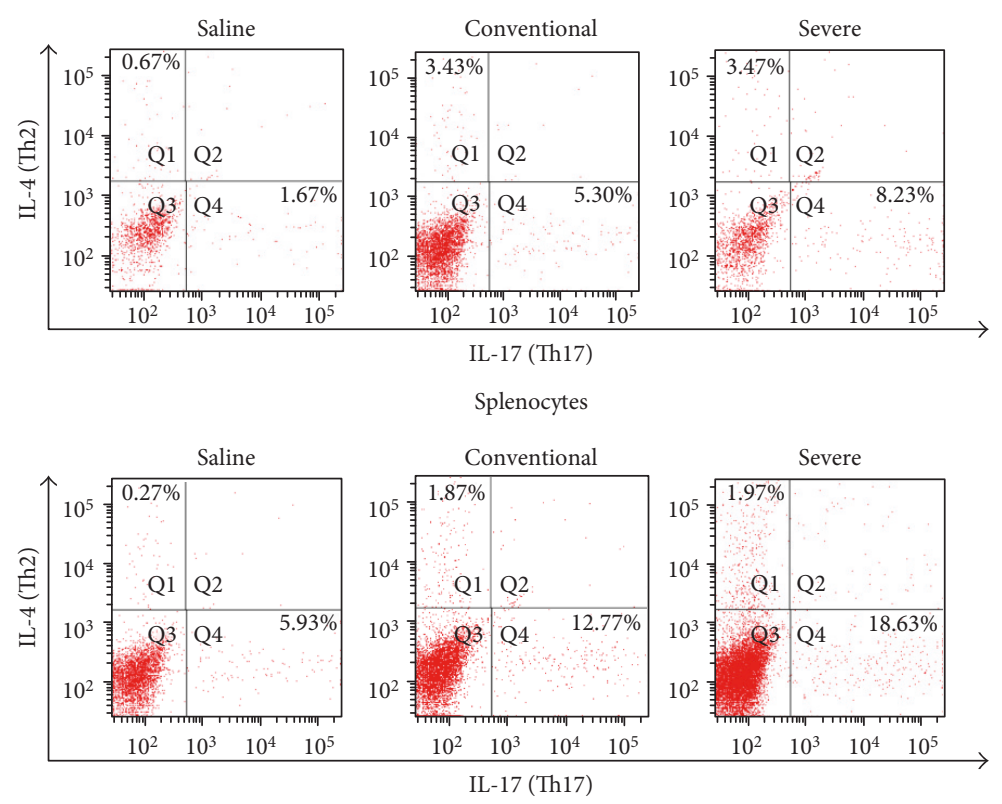

(c)

Figure 2: The severe asthma mice were mediated by Th17 cells. (a) Lung tissues were stained for immunohistochemistry (anti-IL-17, anti-IL-4) of the three groups. Histological analyses of lungs from the severe group exhibited markedly enhanced cells with stained IL-17 positively, but cells with stained IL-4 positively were not obviously different compared with those from the conventional group. (b) Western blot analysis detected IL-17 and IL-4 protein expression in the three groups. The IL-17 protein expression of the lungs from the severe group was higher than that from the conventional group, whereas IL-4 protein expression values were not obviously different between them. (c) Th17 and Th2 cells were tested in bronchial lung tissue suspension cells and splenocytes, and the cells were then subjected to intracellular staining of APC-anti-IL-17 and PE-anti-IL-4 by flow cytometry analyses. Th17 cells were the most in bronchial lung tissue suspension cells and splenocytes from the severe group compared with the conventional group, whereas Th2 cells were not obviously different between them. 

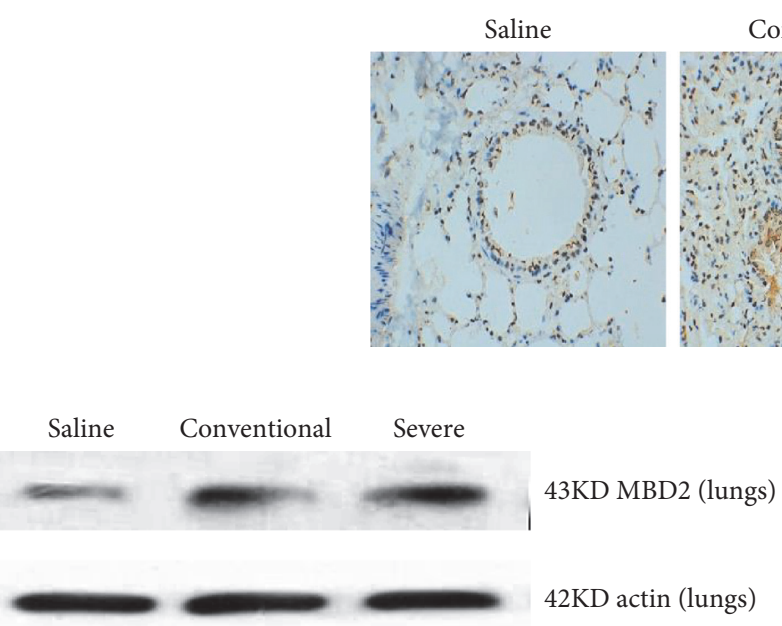

(b)

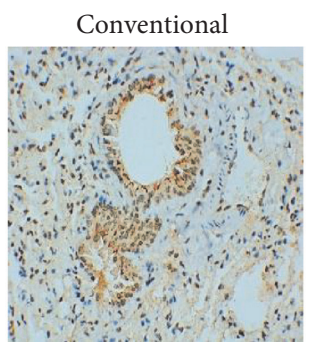

(a)

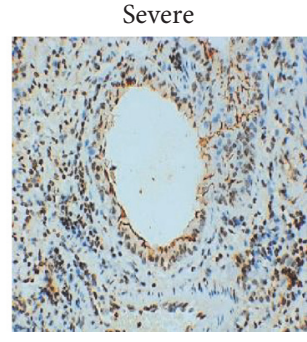

Saline Conventional Severe
43KD MBD2 (splenocytes)

$42 \mathrm{KD}$ actin (splenocytes)

(c)

FIgURE 3: Expression of MBD2 in three groups. (a) Lung tissues were stained for immunohistochemistry (anti-MBD2) of the three groups. Histological analyses of lungs from the severe group exhibited more cells with stained MBD2 positively than those from the conventional group. (b) Western blot analyses detected MBD2 protein expression in the lungs of the three groups. The MBD2 protein expression of the lungs from the severe group was higher than that from the conventional group. (c) Western blot analyses detected MBD2 protein expression in the splenocytes of the three groups. The MBD2 protein expression of the lungs from the severe group was higher than that from the conventional group.

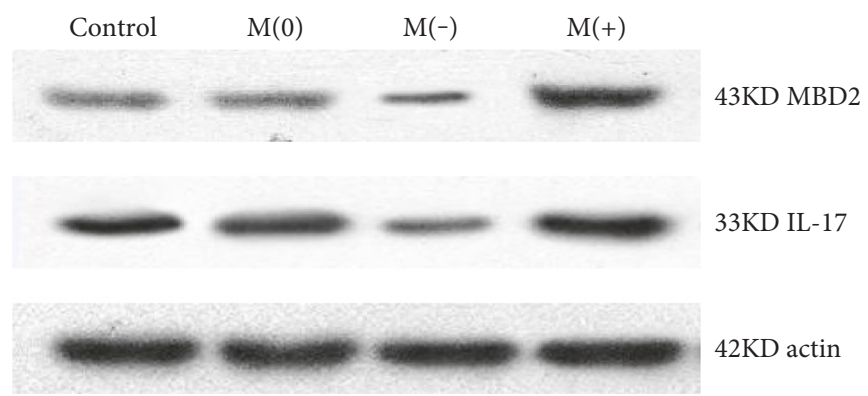

(a)
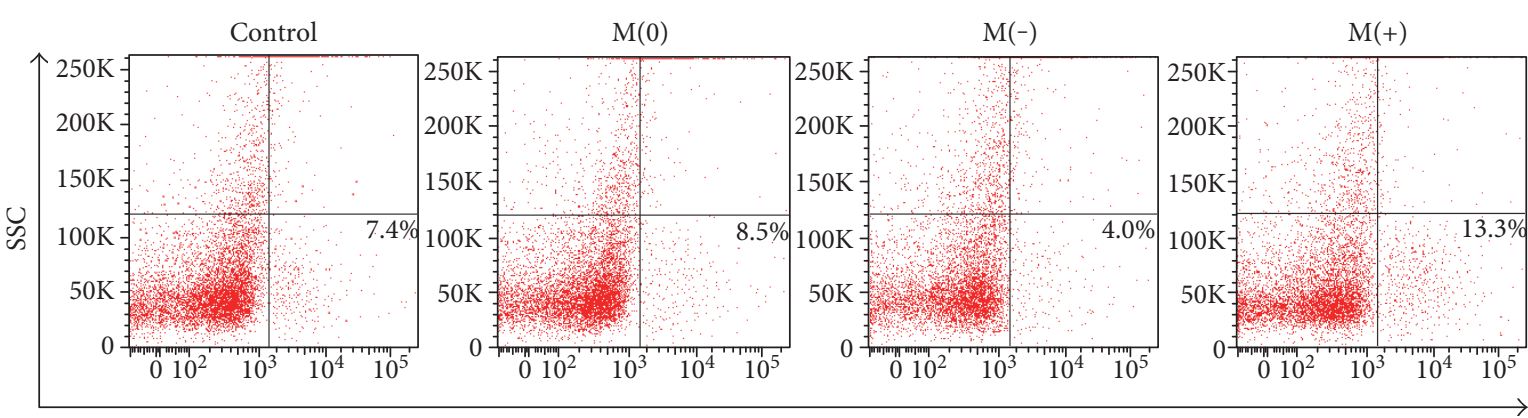

IL-17 (Th17)

(b)

FIGURE 4: IL-17 expression and Th17 cell differentiation under MBD2 gene silencing or overexpression. (a) Under MBD2 gene silencing $(\mathrm{M}(-))$, IL-17 and MBD2 protein expression was significantly lower than that of the empty transfection group (M(0)) by Western blot analyses; with MBD2 gene overexpression $(\mathrm{M}(+))$, IL-17 and MBD2 protein expression was markedly increased than that of $\mathrm{M}(0)$. (b) Under MBD2 gene silencing $(\mathrm{M}(-))$, Th17 cell differentiation was significantly lower than that of $\mathrm{M}(0)$ according to flow cytometry analyses; with MBD2 gene overexpression $(\mathrm{M}(+))$, Th17 cell differentiation was markedly increased compared to that of $\mathrm{M}(0)$. 

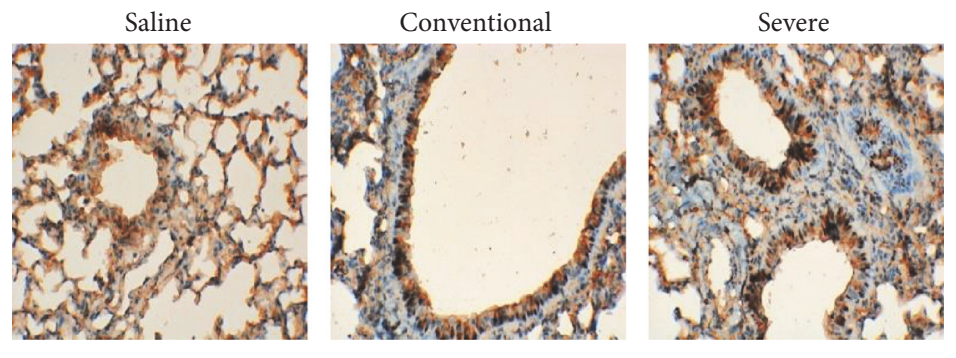

(a)

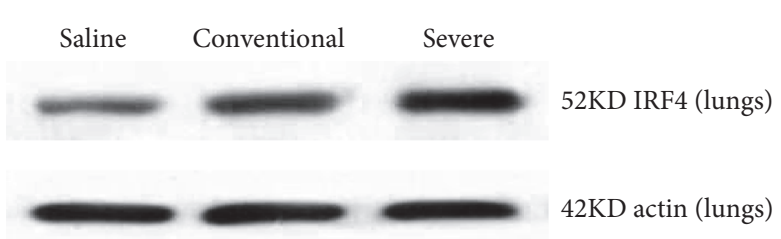

(b)

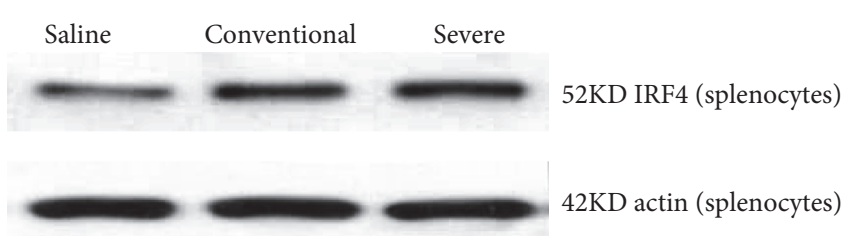

(c)

FIGURE 5: Expression of IRF4 in three groups. (a) Lung tissues were stained for immunohistochemistry (anti-IRF4) of the three groups. Histological analyses of lungs from the severe and conventional groups exhibited markedly enhanced cells with stained IRF4 compared with those of the saline group. (b) Western blot analyses detected IRF4 protein expression in the lungs of the three groups. The IRF4 protein expression from the severe and conventional groups were higher than that from the saline group. (c) Western blot analyses detected IRF4 protein expression in the splenocytes of the three groups. The IRF4 protein expression from the severe and conventional groups were higher than that from the saline group.

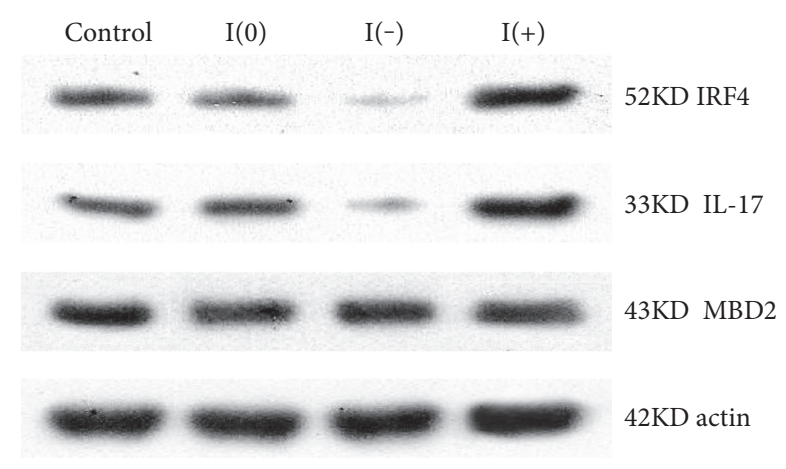

FIGURE 6: IL-17 and MBD2 expression under IRF4 gene silencing $(\mathrm{I}(-))$ or overexpression $(\mathrm{I}(+))$. Under I(-), IL-17 and IRF4 protein expression were significantly lower than that of the empty transfection group $(\mathrm{I}(0))$ according to Western blot analyses; under I(+), IL-17 and IRF4 protein expression were markedly increased compared to that of $\mathrm{I}(0)$. Under $\mathrm{I}(-)$ or $\mathrm{I}(+), \mathrm{MBD} 2$ protein expression showed no significant difference.

3.5. Expression of IRF4 in Severe Asthma Mice. Histological analyses of the lungs from the severe and conventional groups exhibited markedly enhanced cells with stained IRF4 compared with those of the saline group (Figure 5(a)). IRF4 expression in the lungs and splenic CD $4^{+} \mathrm{T}$ cells from the two groups were significantly increased compared with that of the saline group, but there was no significant difference between the two groups (Figures 5(b) and 5(c)).

\subsection{IL-17 and MBD2 Expression under IRF4 Gene Silencing} or Overexpression. We demonstrated that IRF4 gene silencing $(\mathrm{I}(-))$ or overexpression $(\mathrm{I}(+))$ in splenic $\mathrm{CD} 4^{+} \mathrm{T}$ cells was successful. Under IRF4 gene silencing, IL-17 expression was significantly lower than that of the empty transfection group $(\mathrm{I}(0))$, and under IRF4 gene overexpression $(\mathrm{I}(+))$, IL-17 expression was markedly increased compared to that of the empty transfection group. Under IRF4 gene silencing or overexpression, no significant difference in MBD2 expression was observed (Figure 6).

3.7. IL-17 Expression and Th17 Cell Differentiation under Joint MBD2 and IRF4 Gene Silencing or Overexpression. We found that IL-17 expression was significantly the lowest when both MBD2 and IRF4 underwent joint gene silencing, while IL-17 expression was the highest when both MBD2 and IRF4 underwent joint gene overexpression (Figure $7(\mathrm{a})$ ). Th17 cells were obviously the least or the most while both MBD2 and IRF4 underwent joint gene silencing or overexpression (Figure 7(b)).

\section{Discussion}

In order to better study the mechanisms of severe asthma, we urgently need to build a neutrophil-predominant severe asthma model. While OVA is a classic asthma model allergen, exposure to LPS is also known to lead to an increased risk of asthma-like symptoms [17] and the onset of asthma exacerbations $[18,19]$. Meanwhile, the development of allergic asthma is strongly associated with the exposure to HDM $[20,21]$. When OVA-induced asthmatic mice are re-exposed to HDM, the pathomechanism is different from OVA exposure alone [22]. Although there have been lots of similar experiments, there are so little successful severe asthma models. In our previous study, we tried different doses of HDM and OVA combined with $15 \mu \mathrm{g}$ LPS to establish a neutrophil-predominant severe asthma model and found that $100 \mu \mathrm{g} \mathrm{HDM}+100 \mu \mathrm{g}$ OVA $+15 \mu \mathrm{g}$ LPS successfully 


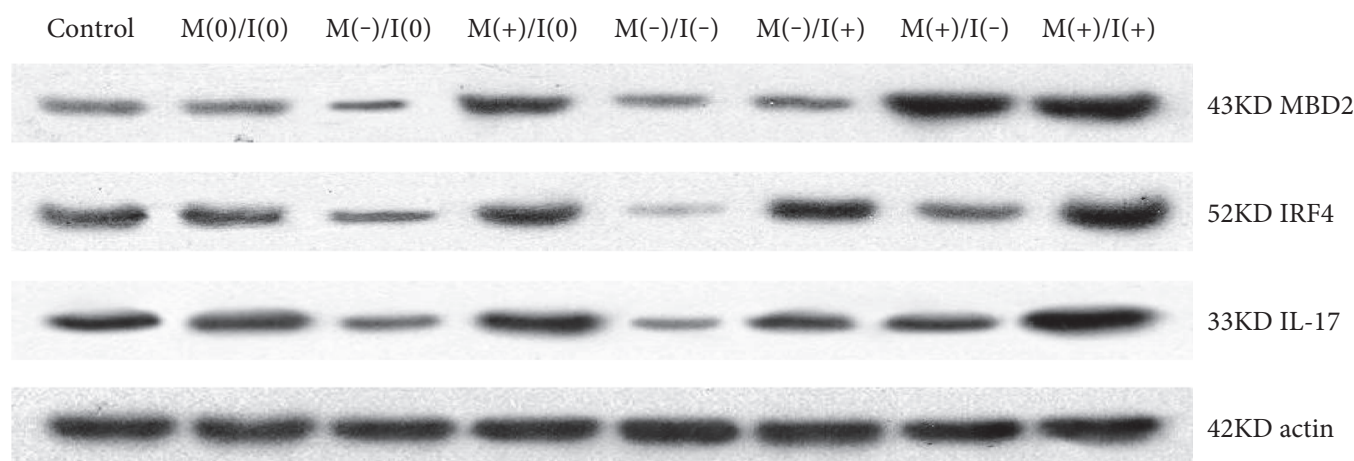

(a)
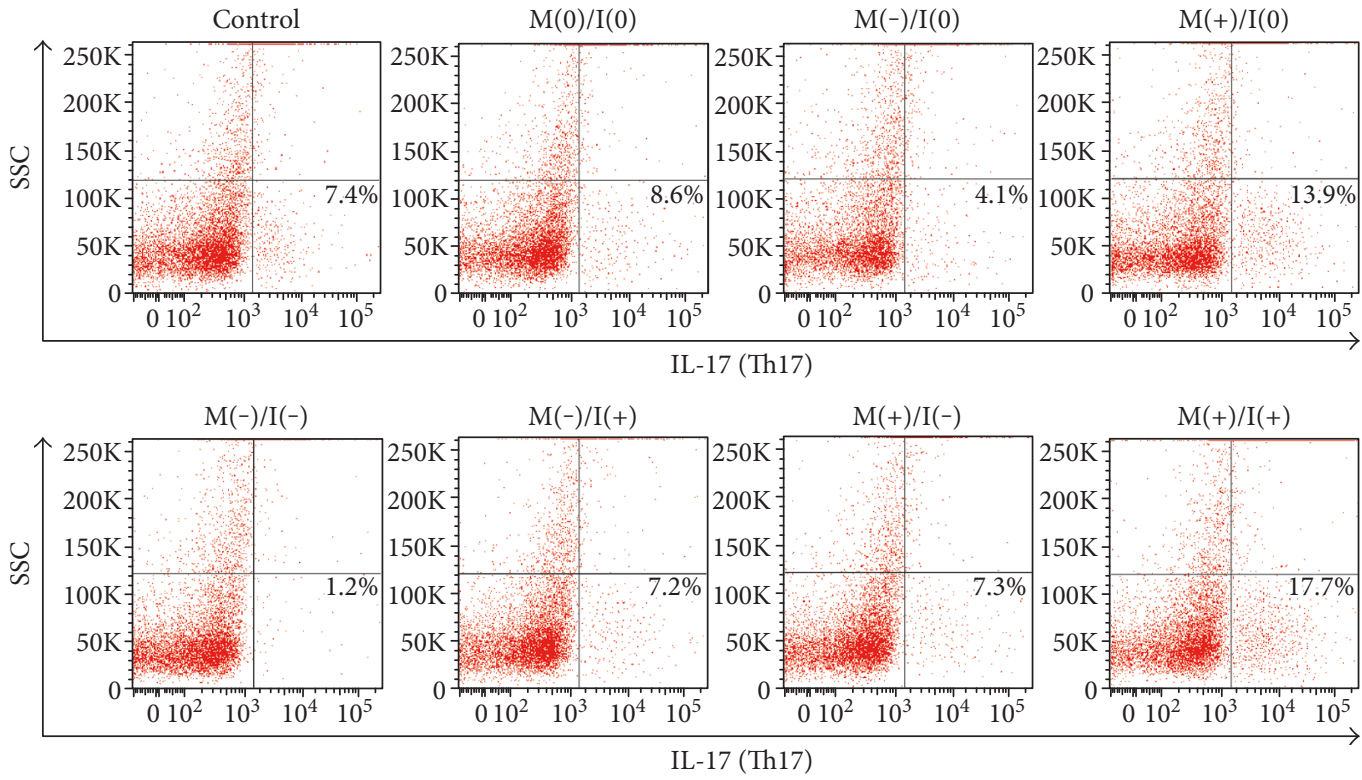

(b)

Figure 7: IL-17 expression and Th17 cell differentiation under joint MBD2 and IRF4 gene silencing or overexpression. (a) Under joint M(-)/ I(-), IL-17 protein expression was significantly the lowest according to Western blot analyses; under joint M(-)/I(0), IL-17 protein expression was higher than $\mathrm{M}(-) / \mathrm{I}(-)$. While under joint $\mathrm{M}(+) / \mathrm{I}(+)$, IL-17 protein expression was significantly the highest; under joint $\mathrm{M}(+) / \mathrm{I}(0)$, IL-17 protein expression was lower than $\mathrm{M}(+) / \mathrm{I}(+)$. IL-17 protein expression showed no significant difference between the control group, $\mathrm{M}(0) / \mathrm{I}(0)$, $\mathrm{M}(-) / \mathrm{I}(+)$, and $\mathrm{M}(+) / \mathrm{I}(-)$. (b) Under joint $\mathrm{M}(-) / \mathrm{I}(-)$, Th17 cell differentiation was significantly the lowest according to flow cytometry analyses; under joint $\mathrm{M}(-) / \mathrm{I}(0)$, Th17 cell differentiation was higher than $\mathrm{M}(-) / \mathrm{I}(-)$. Under joint $\mathrm{M}(+) / \mathrm{I}(+)$, Th17 cell differentiation was significantly the highest; under joint $\mathrm{M}(+) / \mathrm{I}(0)$, Th17 cell differentiation was lower than $\mathrm{M}(+) / \mathrm{I}(+)$. No significant difference in Th17 cell differentiation was observed between the control group, $\mathrm{M}(0) / \mathrm{I}(0), \mathrm{M}(-) / \mathrm{I}(+)$, and $\mathrm{M}(+) / \mathrm{I}(-)$.

established a NEU predominant inflammatory phenotype severe asthma model. Here, compared with the conventional asthma mice sensitized by OVA only (named the OVA group), among BALF total cell counts, BALF NEU was significantly increased in the severe asthma mice sensitized by HDM/OVA/LPS, but BALF EOS showed an opposite tendency. Similarly, analysis of lung tissue sections revealed that the HDM/OVA/LPS sensitized group caused a significant increase of both histological inflammation scoring and NEU expression compared to the OVA group, whereas there was no difference in the expression of EOS between the two groups. Compared to moderate asthma, IL-4 expression was decreased [7] and IL-17A expression was increased [23, 24] in severe asthma. In the present study, lung tissue IL17A expression was significantly increased in the HDM/
OVA/LPS sensitized group when compared with the OVA group, whereas there was no difference in the expression of IL-4 between the two groups. The spleen is the classical observation target for immune cells, and Th17 cells were the most in the splenocytes from severe asthma mice compared with conventional asthma mice according to flow cytometry. The lung is one of the effector organs of immunity, and Th17 cells were the most in the lung tissue suspension cells from severe asthma mice compared with conventional asthma mice according to flow cytometry. This showed that neutrophilic predominant severe asthma mice first exhibited mainly Th17 cell differentiation followed by an increase of IL-17 secretion, and the recruitment of NEU, thus promoting the development of asthma, in a way distinctly different from the classic EOS allergic asthma 
involving IL-4 recruitment. Therefore, the neutrophilpredominant severe asthma model was established successfully.

MBD2, as an epigenetic regulation element [25], can regulate $\mathrm{T}$ cells to differentiate into Th17 cells by methylating Tbet/Hlx [26], so it could be involved in the pathogenesis of severe asthma. Considering that Th17 cell is mainly involved in the inflammatory response, after being induced by oligodendrocytes, EAE mice would develop severe paralysis, but MBD2 null mutant mice were completely protected from EAE induction [26]. In our study, MBD2 showed significantly increased expression in the splenocytes of the severe asthma model mice, representing the classical observation target as immune cells. The lung is one of the effector organs of immunity, and MBD2 showed a higher expression in the lung tissue of severe asthma model mice compared with the conventional asthma model mice. To ascertain whether MBD2 is involved in the pathogenesis of severe asthma, we conducted in vitro splenocyte experiments and found that IL-17 protein expression increased significantly along with overexpression of the MBD2 gene and decreased with the silencing of the MBD2 gene. At the same time, the number of Th17 cells showed a consistent change with the overexpression or silencing of the MBD2 gene. Through these tests, MBD2 is shown to participate in severe asthma by affecting the differentiation of Th17 cells and IL-17 secretion.

Next, we needed to analyze whether MBD2 can affect IRF4 and participate in asthma. IRF4, as an essential controller of Th17 differentiation, participates in many immune diseases [27]. IRF4-deficient mice are totally resistant to the development of a Th17-mediated disease [10]. Overexpressed IRF4 in naive $\mathrm{CD}^{+} \mathrm{T}$ cells derived from relapsing remitting multiple sclerosis patients significantly increased their ability to secrete IL-17A, IL-17F, IL-21, and IL-22 [28]. We found that both IRF4 and IL-17 had a significantly increased expression in the lung and the spleen cells of severe asthma model mice. From the in vitro cell experiment, IL-17 protein expression increased significantly with overexpression of the IRF4 gene and decreased with the silencing of the IRF4 gene. These findings show that IRF4 participates in severe asthma by affecting the differentiation of IL-17 secretion and is consistent with previous studies. Then, from the in vitro splenocyte experiment, we found that IRF4 protein expression increased significantly along with overexpression of the MBD2 gene and decreased with the silencing of the MBD2 gene, whereas there was no significant difference in the expression of MBD2 under overexpression or silencing of the IRF4 gene. This shows that MBD2 can affect IRF4 expression in severe asthma.

Finally, in conditions of joint overexpression of both MBD2 and IRF4 genes, the levels of expression of IRF4, differentiation of Th17 cells, and IL-17 secretion were the highest, while joint silencing of MBD2 and overexpression of IRF4 genes, the levels of expression of IRF4, differentiation of Th17 cells, and IL-17 secretion were declined. Under conditions of joint silencing of both genes, the levels of expression of IRF4 and differentiation of Th17 cells and IL17 secretion were the lowest, while joint overexpression of MBD2 and silencing of IRF4 genes, the levels of expression of IRF4, and differentiation of Th17 cells and IL-17 secretion were increased. This indicates that MBD2 can participate in the differentiation of Th17 cells and IL-17 secretion through IRF4 expression and so participate in the pathogenesis of the neutrophil-predominant severe asthma model.

\section{Abbreviations \\ MBD2: Methyl-CpG binding domain protein 2 \\ BALF: Bronchoalveolar lavage fluid \\ AHR: Airway hyperresponsiveness \\ IRF4: Interferon regulatory factor 4 \\ HDM: House dust mite \\ OVA: Ovalbumin \\ LPS: Lipopolysaccharide \\ NEU: Neutrophil granulocyte \\ EOS: Eosinophilic granulocyte \\ RL: Pulmonary resistance.}

\section{Conflicts of Interest}

The authors report no conflicts of interest.

\section{Authors' Contributions}

Yueling Wang and Aijun Jia contributed equally to this work.

\section{Acknowledgments}

The authors thank Professor Cong-Yi Wang, Center for Biomedical Research, Tongji Hospital, Huazhong University of Science and Technology, China, for the data analysis and guiding the manuscript writing. This study was supported by the National Natural Science Foundation of China (81370128) and the Natural Science Foundation of Hunan Province (14JJ2028).

\section{References}

[1] M. Patyk, A. Obojski, Ł. Gojny, B. Panaszek, and U. ZaleskaDorobisz, "Airway evaluation with multidetector computed tomography post-processing methods in asthmatic patients," Advances in Experimental Medicine and Biology, vol. 934, pp. 41-47, 2016.

[2] L. Qin, J. Feng, C. Hu, Y. Li, and R. Niu, "Th17/Treg imbalance mediated by IL-8 in RSV-infected bronchial epithelial cells," Zhong nan da Xue Xue Bao. Yi Xue Ban=Journal of Central South University. Medical sciences, vol. 41, no. 4, pp. 337344, 2016.

[3] J. M. Rumble, A. K. Huber, G. Krishnamoorthy et al., "Neutrophil-related factors as biomarkers in EAE and MS," The Journal of Experimental Medicine, vol. 212, no. 1, pp. 23-35, 2015.

[4] W. Xu, L. Chen, S. Guo, L. Wu, and J. Zhang, "Intranasal administration of recombinant mycobacterium smegmatis inducing IL-17A autoantibody attenuates airway inflammation in a murine model of allergic asthma," PLoS One, vol. 11, no. 3, article e0151581, 2016.

[5] M. Berry, A. Morgan, D. E. Shaw et al., "Pathological features and inhaled corticosteroid response of eosinophilic and non- 
eosinophilic asthma," Thorax, vol. 62, no. 12, pp. 1043-1049, 2007.

[6] J. Douwes, P. Gibson, J. Pekkanen, and N. Pearce, "Non-eosinophilic asthma: importance and possible mechanisms," Thorax, vol. 57, no. 7, pp. 643-648, 2002.

[7] A. T. Hastie, W. C. Moore, D. A. Meyers et al., "Analyses of asthma severity phenotypes and inflammatory proteins in subjects stratified by sputum granulocytes," The Journal of Allergy and Clinical Immunology, vol. 125, no. 5, pp. 1028.e131036.e13, 2010.

[8] X. M. Li, J. Peng, W. Gu, and X. J. Guo, "TCDD-induced activation of aryl hydrocarbon receptor inhibits Th17 polarization and regulates non-eosinophilic airway inflammation in asthma," PLoS One, vol. 11, no. 3, article e0150551, 2016.

[9] J. Shannon, P. Ernst, Y. Yamauchi et al., "Differences in airway cytokine profile in severe asthma compared to moderate asthma," Chest, vol. 133, no. 2, pp. 420-426, 2008.

[10] A. Brüstle, S. Heink, M. Huber et al., "The development of inflammatory $\mathrm{T}(\mathrm{H})-17$ cells requires interferon-regulatory factor 4," Nature Immunology, vol. 8, no. 9, pp. 958-966, 2007.

[11] J. Daan de Boer, J. J. Roelofs, A. F. de Vos et al., "Lipopolysaccharide inhibits Th2 lung inflammation induced by house dust mite allergens in mice," American Journal of Respiratory Cell and Molecular Biology, vol. 48, no. 3, pp. 382-389, 2013.

[12] S. M. Hoffman, J. E. Tully, J. D. Nolin et al., "Endoplasmic reticulum stress mediates house dust mite-induced airway epithelial apoptosis and fibrosis," Respiratory Research, vol. 14, p. 141, 2013.

[13] N. R. Locke, S. G. Royce, J. S. Wainewright, C. S. Samuel, and M. L. Tang, "Comparison of airway remodeling in acute, subacute, and chronic models of allergic airways disease," American Journal of Respiratory Cell and Molecular Biology, vol. 36, no. 5, pp. 625-632, 2007.

[14] H. S. Bandukwala, B. S. Clay, J. Tong et al., "Signaling through Fc gamma RIII is required for optimal T helper type (Th)2 responses and Th2-mediated airway inflammation," The Journal of Experimental Medicine, vol. 204, no. 8, pp. 18751889, 2007.

[15] G. Hu, Q. Tang, S. Sharma et al., "Expression and regulation of intergenic long noncoding RNAs during T cell development and differentiation," Nature Immunology, vol. 14, no. 11, pp. 1190-1198, 2013.

[16] Y. H. Wang, K. S. Voo, B. Liu et al., "A novel subset of CD4(+) $\mathrm{T}(\mathrm{H}) 2$ memory/effector cells that produce inflammatory IL-17 cytokine and promote the exacerbation of chronic allergic asthma," The Journal of Experimental Medicine, vol. 207, no. 11, pp. 2479-2491, 2010.

[17] L. A. Smit, D. Heederik, G. Doekes, C. Blom, I. van Zweden, and I. M. Wouters, "Exposure-response analysis of allergy and respiratory symptoms in endotoxin-exposed adults," The European Respiratory Journal, vol. 31, no. 6, pp. 1241-1248, 2008.

[18] G. D. Kang and D. H. Kim, "Poncirin and its metabolite ponciretin attenuate colitis in mice by inhibiting LPS binding on TLR4 of macrophages and correcting Th17/Treg imbalance," Journal of Ethnopharmacology, vol. 189, pp. 175$185,2016$.

[19] H. Tan, P. Pan, L. Zhang et al., "Nerve growth factor promotes expression of costimulatory molecules and release of cytokines in dendritic cells involved in Th2 response through LPS-induced p75NTR," The Journal of Asthma, vol. 53, no. 10, pp. 989-998, 2016.

[20] C. Huang, H. Dong, M. Zou et al., "Bevacizumab reduced autophosphorylation of VEGFR2 to protect HDM-induced asthma mice," Biochemical and Biophysical Research Communications, vol. 478, no. 1, pp. 181-186, 2016.

[21] K. Shima, T. Koya, K. Tsukioka et al., "Effects of sublingual immunotherapy in a murine asthma model sensitized by intranasal administration of house dust mite extracts," Allergology International, vol. 66, no. 1, pp. 89-96, 2017.

[22] F. Lan, K. Liu, J. Zhang, Y. Qi, K. Li, and P. Lin, "Th17 response is augmented in OVA-induced asthmatic mice exposed to HDM," Medical Science Monitor, vol. 17, no. 5, pp. BR132BR138, 2011.

[23] M. Zhang, G. Nong, M. Jiang, and W. Zhan, "Lipid derivative of benzylidene malononitrile AG490 attenuates airway inflammation of mice with neutrophilic asthma," Xi Bao Yu fen $\mathrm{Zi}$ Mian Yi Xue Za Zhi=Chinese journal of cellular and molecular immunology, vol. 32, no. 6, pp. 730-733, 2016.

[24] Z. Zhang, J. M. Biagini Myers, E. B. Brandt et al., "Beta-glucan exacerbates allergic asthma independent of fungal sensitization and promotes steroid-resistant TH2/TH17 responses," The Journal of Allergy and Clinical Immunology, vol. 139, no. 1, pp. 54.e8-65.e8, 2017.

[25] K. H. Wood, B. S. Johnson, S. A. Welsh et al., "Tagging methylCpG-binding domain proteins reveals different spatiotemporal expression and supports distinct functions," Epigenomics, vol. 8, no. 4, pp. 455-473, 2016.

[26] J. Zhong, Q. Yu, P. Yang et al., "MBD2 regulates TH17 differentiation and experimental autoimmune encephalomyelitis by controlling the homeostasis of T-bet/Hlx axis," Journal of Autoimmunity, vol. 53, pp. 95-104, 2014.

[27] A. Nalbant and D. Eskier, "Genes associated with T helper 17 cell differentiation and function," Frontiers in Bioscience (Elite Edition), vol. 8, pp. 427-435, 2016.

[28] X. Zhang, Y. Tao, L. Troiani, and S. Markovic-Plese, "Simvastatin inhibits IFN regulatory factor 4 expression and Th17 cell differentiation in CD4+ $\mathrm{T}$ cells derived from patients with multiple sclerosis," Journal of Immunology, vol. 187, no. 6, pp. 3431-3437, 2011. 


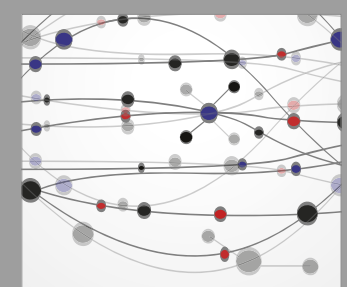

The Scientific World Journal
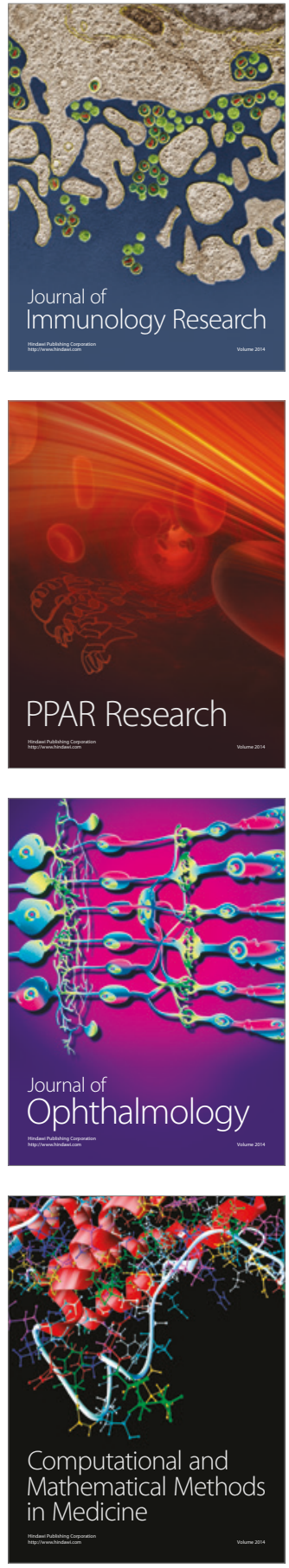

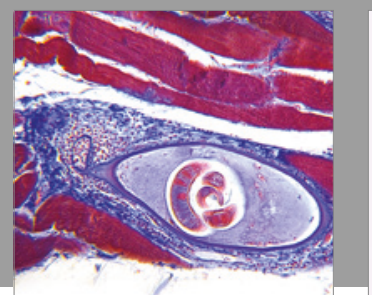

Gastroenterology Research and Practice
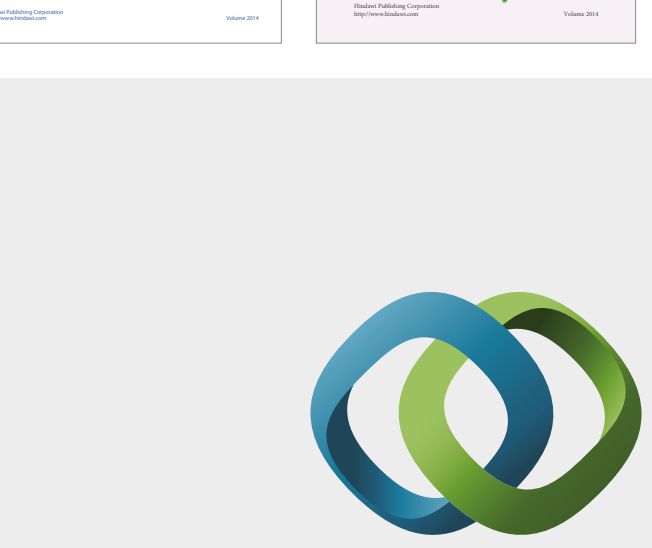

\section{Hindawi}

Submit your manuscripts at

https://www.hindawi.com
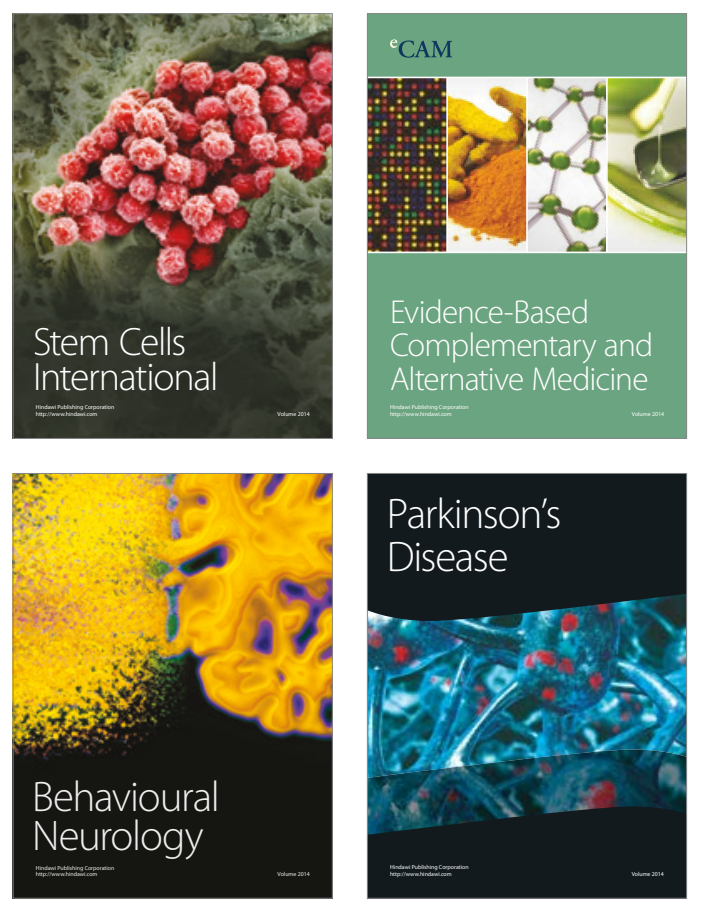
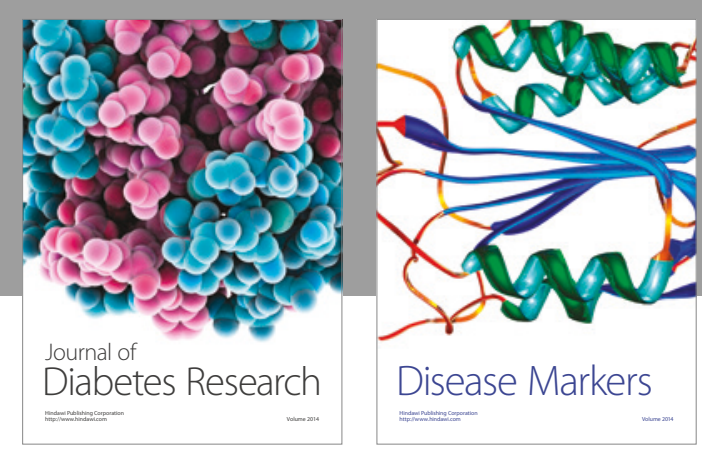

Disease Markers
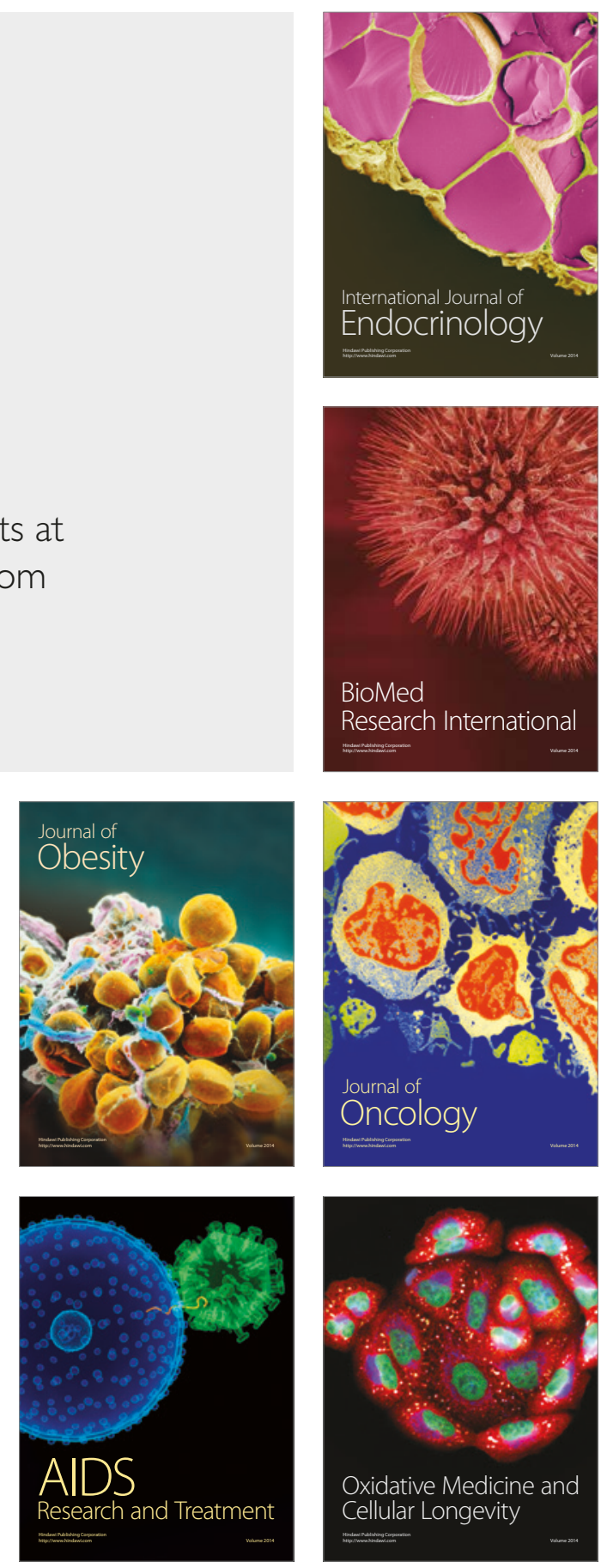